\title{
Dexamethasone Intracanalicular Insert: A Review in Treating Post-Surgical Ocular Pain and Inflammation
}

\author{
Arnold Lee ${ }^{1}\left[\right.$ Hannah A. Blair ${ }^{1}$ \\ Published online: 25 June 2020 \\ ○) Springer Nature 2020, corrected publication 2020
}

\begin{abstract}
Dextenza $^{\circledR}$, an intracanalicular insert that is placed into the lower punctal opening of the eye, gradually releases dexamethasone for up to 30 days to alleviate pain and inflammation associated with ophthalmic surgery. A significantly higher proportion of patients treated with the dexamethasone intracanalicular insert than with the placebo insert had no pain at day 8 (co-primary endpoint, 7 days post-operation) across three pivotal phase III trials, and the inflammation co-primary endpoint (absence of anterior chamber cells) at day 14 (13 days post-operation) was met in two of three trials. Overall, the dexamethasone intracanalicular insert was effective and generally well tolerated for the treatment of post-surgical ocular pain and inflammation following cataract surgery. As low patient adherence is an issue for topical ophthalmic anti-inflammatory medications, the convenience (ease of insertion, single application with no patient input and typically no removal required) of the dexamethasone intracanalicular insert makes it a promising emerging option for the treatment of ocular inflammation and pain following ophthalmic surgery.
\end{abstract}

\section{Dexamethasone intracanalicular insert: clinical considerations}

A single insert placed by an ophthalmologist results in tapered release of $0.4 \mathrm{mg}$ dexamethasone for up to 30 days

Reduces ocular pain and inflammation following cataract surgery

Manual expression of the insert is not typically required as the insert is resorbable

Generally well tolerated; high patient satisfaction rates

Enhanced material for this Adis Drug Evaluation can be found at https://doi.org/10.6084/m9.figshare.12344354.

The manuscript was reviewed by: $A$. L. Robin, Department of Ophthalmology, University of Michigan, Ann Arbor, MI, USA; Department of Ophthalmology and International Health, Johns Hopkins University, Baltimore, MD, USA; M. K. Rhee, Department of Ophthalmology, Icahn School of Medicine at Mt Sinai, New York, NY, USA.

Electronic supplementary material The online version of this article (https://doi.org/10.1007/s40265-020-01344-6) contains supplementary material, which is available to authorized users.

Arnold Lee

demail@ springer.com

1 Springer Nature, Private Bag 65901, Mairangi Bay, Auckland 0754, New Zealand

\section{Introduction}

The demand for ophthalmic surgeries is high, with millions of cataract surgeries (the most common ophthalmic surgery) performed annually in the USA [1]. In addition to cataract surgeries, demand exists for other ophthalmic surgeries, with approximately 800,000 refractive surgeries in 2010 and approximately 48,000 corneal transplants in 2013 in the USA [2].

Corticosteroid eye drops may be prescribed following ophthalmic surgery, as inflammation is a risk factor for complications such as pain [3] and conditions associated with visual degradation such as cystoid macular oedema [3] or posterior capsule opacification [4] following cataract surgery. However, adherence with corticosteroid eye drops may decrease due to the dosing frequency (multiple administrations daily) [3], and may be exacerbated by generally poor administration techniques with eye drops, as seen in an observational study $(\approx 20-30 \%$ of glaucoma patients successfully instilled a drop without touching the tip of the bottle) [5]. Poor adherence with ophthalmic preparations is an important contributor to treatment failure [3], thus, there is a need to simplify post-surgical treatment regimens.

Dexamethasone ophthalmic insert $0.4 \mathrm{mg}$ (Dextenza ${ }^{\circledR}$; hereafter referred to as the dexamethasone intracanalicular insert, or dexamethasone insert) is an FDA-approved insert that delivers a tapered dosage of dexamethasone for up to 30 days after surgery without patient input or handling [6]. This review 
provides an overview of the pharmacology, efficacy and tolerability of the dexamethasone intracanalicular insert for the treatment of post-surgical ocular pain and inflammation.

\section{Pharmacological Properties of the Dexamethasone Insert}

The mechanism of action of dexamethasone is well established [7] and is summarized in Table 1. However, the pharmacokinetic properties are unique to the dexamethasone intracanalicular insert in comparison with other formulations, in particular due to the extended release of dexamethasone over 28 days (Table 1, Sect. 2.1). Systemic absorption of dexamethasone was minimal in healthy human volunteers (Table 1), thus complete pharmacokinetic information is unavailable due to low systemic exposure [8]. The insert was retained for 30 days in most patients in phase III trials (Sect. 3), and is expected to be cleared from the canaliculus without the need for removal by manual expression (Table 1).

Investigators determined the dexamethasone intracanalicular insert was easy to administer in the majority of patients and easy or moderately easy to remove (when required) during phase III trials (Table 1). Furthermore, visualization of the dexamethasone intracanalicular insert was deemed to be easy in most patients (Table 1), as the fluorescent insert may be viewed with a yellow filter when the area is illuminated with blue light [6]. However, as visualisation was difficult with certain skin tones, retention rates are considered to be conservative estimates [9].

\subsection{Release Properties}

Dexamethasone was released from the insert over 28 days with a tapered release profile (Table 1). The release rate was dependent on the solubility of dexamethasone in tear fluid, as the release rate of poorly soluble corticosteroids appeared to be proportional to their aqueous solubility in canine models [10]. Furthermore, the release of dexamethasone from the insert occurred primarily from the face of the insert that was closest to the punctal opening due to tear fluid lavage [10]. The release rate of dexamethasone was unaffected by the amount of dexamethasone loading over a dose range of 215-718 $\mu \mathrm{g}$, with greater loading amounts corresponding to a longer duration of release [11].

\section{Therapeutic Efficacy of the Dexamethasone Insert}

The therapeutic efficacy of the dexamethasone intracanalicular insert for the treatment of post-surgical ocular pain and inflammation was demonstrated in three randomized, double-blind, phase III trials [OTX-13-002 $(n=247)$ [12], OTX-14-003 $(n=241)$ [12] and OTX-15-003 $(n=438)$ [13]; Fig. 1]. These pivotal trials were supported by a smaller phase II trial $(n=60)$ [14]. In the phase III trials, dexamethasone $0.4 \mathrm{mg}$ or placebo intracanalicular inserts (i.e. an insert without dexamethasone) were placed during cataract surgery

\section{Table 1 Pharmacology and other properties of the dexamethasone intracanalicular insert}

Pharmacodynamic properties of dexamethasone

Mechanism of action Binds to the glucocorticoid receptor in the cytoplasm (equilibrium dissociation constant 11.25 nmol/L [33]) resulting in upregulation of anti-inflammatory messengers (e.g. annexin I) and downregulation of pro-inflammatory messengers (e.g. NF- $\mathrm{kB}$ ), ultimately leading to a decrease in inflammatory prostaglandins [7]

Decreases inflammatory processes (e.g. oedema, recruitment of inflammatory cells, leakage of capillaries and fibrin deposition) [6]

Pharmacokinetic properties of the dexamethasone intracanalicular insert

Release properties $\quad$ Tapered release from dexamethasone $0.4 \mathrm{mg}$ inserts occurred over 28 days (median $267 \mu \mathrm{g}$ dexamethasone remaining in explanted inserts on day 7 and tapered to $0 \mu \mathrm{g} / \mathrm{mL}$ by day 28) in a canine model [34]

Systemic absorption Very limited systemic absorption of dexamethasone occurred in humans (plasma samples from 5/16 healthy volunteers were below lower LOQ at all time points, $\mathrm{C}_{\max }$ was $<1 \mathrm{ng} / \mathrm{mL}$ ) [35]

Other properties of the dexamethasone intracanalicular insert

Retention and clearance The insert was retained for 30 days in $90.4 \%$ of 530 pts across three phase III trials [9]

Hydrolysis of the PEG polymer leads to degradation and clearance of the insert; removal via manual expression or irrigation with saline is not required [12]

Ease of use Placement of the dexamethasone insert was deemed to be easy by investigators in $76.5 \%$ of pts across three phase III trials $(n=541)$ [36] and, when required, removal was reported to be easy or moderately easy in all removals of dexamethasone $(n=2)$ or placebo $(n=7)$ inserts [9]

The fluorescent insert was easily visualized by investigators in $94.0 \%$ of pts on day 2 (day 1 post-operation) and $83.3 \%$ of pts in phase III trials by day $30[9,36]$

$C_{\text {max }}$ maximum plasma concentration, $L O Q$ limit of quantification, $P E G$ polyethylene glycol, pts patients 
Fig. 1 Design of phase III trials (OTX-13-002 [12], OTX-14003 [12] and OTX-15-003 [13]) evaluating the efficacy of the dexamethasone intracanalicular insert in post-surgical ocular pain and inflammation as assessed by the absence of AC cells, with the proportion of patients achieving an absence of pain or AC cells reported in the animated figure (available online). Dotted outlines indicate follow-up visits if the insert is detected on day $60 . * p \leq 0.05$ vs placebo in the respective trial. $A C$ anterior chamber

\section{OTX-13-002 OTX-14-003}

Evaluation

Follow-up

Dexamethasone insert
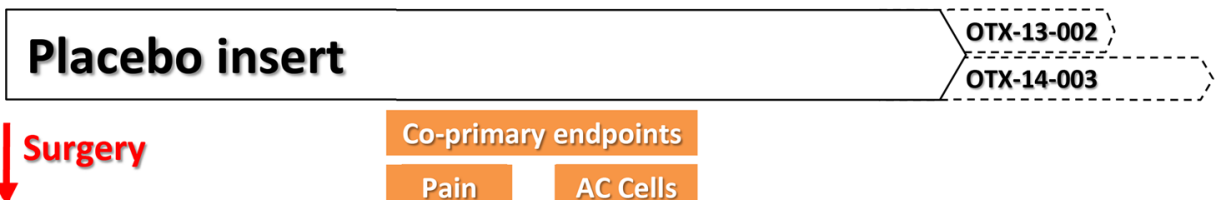

$1-2-4-8-30-60-90-120$

Days

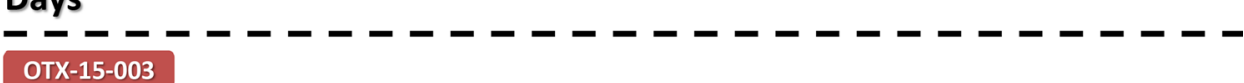

Evaluation

Dexamethasone insert

\section{Placebo insert}

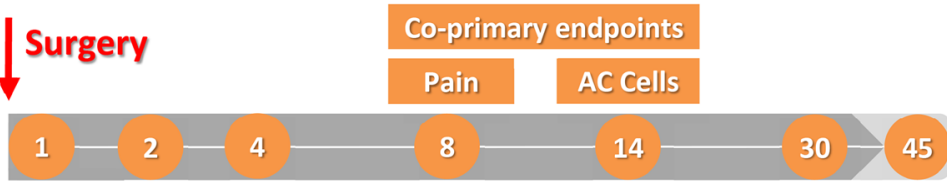

Days

on day 1 of the study (Fig. 2), and patients attended clinic visits up to day 120 in OTX-13-002, day 90 in OTX-14-003 and day 45 in OTX-15-003 (Fig. 1).

Inclusion criteria were patients with cataracts who were aged $\geq 18$ years, and were scheduled to undergo clear corneal cataract surgery with phacoemulsification and implantation of intraocular lens. Patients were required to have a predicted post-surgical Snellen visual acuity of 20/200 or better in both eyes. Key exclusion criteria included corneal or retinal surgery or procedure within the previous 6 months or planned during the trial period, pre-existing ocular pain, macular disease (or oedema in OTX-13-002 and OTX-14003), presence of ocular inflammation, history of or pharmaceutical treatment for glaucoma or ocular hypertension and spikes in intraocular pressure (IOP) in either eye [12, 13]. The use of topical non-steroidal anti-inflammatory drugs (NSAIDs) was restricted to rescue therapy at the discretion of the investigator, however, the use of rescue medication was considered as treatment failure. Systemic NSAIDs were permitted [12], but were restricted to $375 \mathrm{mg} /$ day in OTX15-003 [13]. The mean age of patients was 67.3-69.9 years across all treatment and placebo groups in the phase III trials, $48.8-62.8 \%$ of patients were female $[12,13]$ and $31.5 \%$ of patients received cataract surgery for their second eye [15].

The co-primary endpoints across all trials were the absence of ocular pain on day 8 (7 days post-operation), and the absence of cells in the anterior chamber (AC) of the eye (indicating inflammation) on day 14 (Fig. 1; 13 days postoperation). Pain was evaluated using an 11 point scale where 0 indicates the absence of pain, and the AC cell score was graded by Standardization of Uveitis Nomenclature Working Group criteria, a $0-4$ scale where a grade of 0 indicates zero cells $[12,13]$. Hierarchical testing order for the co-primary endpoints in OTX-13-002 and OTX-14-003 was the absence of AC cells and then the absence of pain [16].

The dexamethasone intracanalicular insert was superior to placebo for the treatment of post-surgical pain, and generally superior to placebo for post-surgical inflammation (Table 2). In all three trials, a significantly higher percentage of patients treated with the dexamethasone intracanalicular insert reported the absence of ocular pain on day 8 (co-primary endpoint) than placebo-treated patients (Table 2). The proportion of patients with the absence of AC cells on day 14 (co-primary endpoint) was significantly higher in the dexamethasone treatment group in 


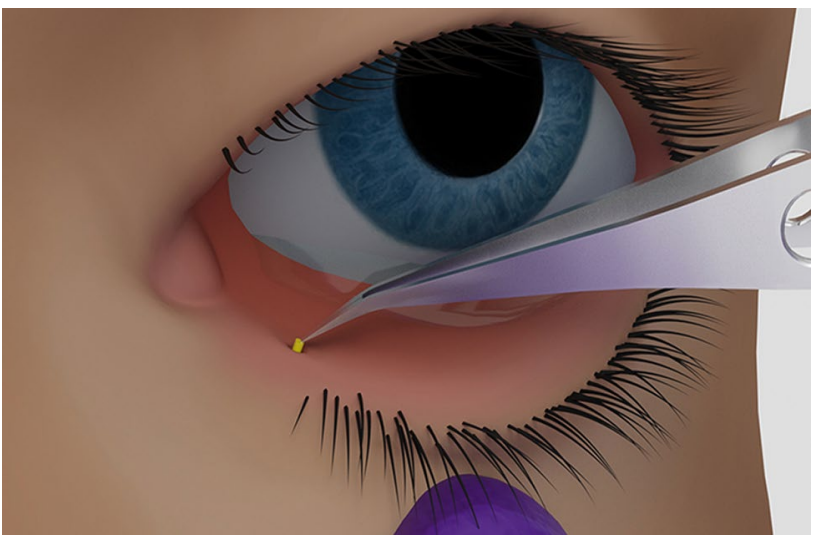

Fig. 2 Placement of the dexamethasone intracanalicular insert through the lacrimal punctum and into the lower canaliculus. Reproduced with permission from Ocular Therapeutix, Inc

comparison to the placebo group in two of three trials (Table 2) [12, 13].

The superiority of the dexamethasone intracanalicular insert to placebo was also demonstrated at other time points. Across all trials, a significantly $(p<0.01)$ higher percentage of patients reported the absence of pain from day 2 to day 14 in the dexamethasone intracanalicular insert groups than the placebo groups $[12,13]$. In the OTX-13-002 and OTX-14-003 trials this significant difference in ocular pain was maintained for all visits to day $60(p<0.01)$ [17]; and in OTX-15-003 significant differences in ocular pain were maintained to day 30 (89.1\% dexamethasone intracanalicular insert vs $80.0 \%$ placebo; $p=0.0095$ ), but not on day 45 due to gradual improvement over time in the placebo group (90.6\% vs $86.5 \%$ ) [13]. The proportion of patients with the absence of AC cells was reported for days 2-45 in OTX-15-003, where no significant differences across the treatment groups were reported on day $2(1.9 \%$ vs $2.3 \%)$, however, at subsequent visits on days $4-45$, a significantly higher $(p<0.05)$ proportion of patients in the dexamethasone intracanalicular insert group achieved an absence of AC cells [13]. Significant differences in the absence of AC cells for OTX-13-002 was maintained between days 14-60 $(p<0.0001)$, and a significant difference was detected on day 60 for OTX-14-003 (73.0\% vs 50.7\%; $p=0.0012)$ [17].

The efficacy of the dexamethasone intracanalicular insert for the treatment of post-surgical inflammation was supported by the absence of AC flare (Table 2) [secondary endpoint]. A significantly higher proportion of patients treated with the dexamethasone intracanalicular insert than placebo had an absence of AC flare across all trials on both day 8 and 14 (Table 2), which was also observed in OTX-15-003 on days 2-30. Gradual improvements in the placebo group resulted in comparable results across the treatment groups in OTX-15-003 on day 45 (92.6\% vs $87.5 \%$ ) [13]. AC flare was graded using Standardization of Uveitis Nomenclature Working Group criteria, a $0-4$ scale where a grade of 0 indicates the absence of flare [12,13].

Patients treated with the dexamethasone intracanalicular insert required less rescue medication than placebo-treated patients (Table 2). In OTX-13-002 and OTX-14-003, a significantly smaller proportion of patients in the dexamethasone intracanalicular insert group than the placebo group required rescue therapy on days 8 and 14 (Table 2). At earlier timepoints (days 1-4) the use of rescue medications was comparable between the dexamethasone intracanalicular insert and placebo groups across all three trials $[12,13]$.

Table 2 Efficacy of dexamethasone intracanalicular insert in post-surgical ocular pain and inflammation in pivotal phase III trials

\begin{tabular}{|c|c|c|c|c|c|c|c|c|c|}
\hline \multirow[t]{2}{*}{ Trial } & \multirow[t]{2}{*}{$\begin{array}{l}\text { Treatment } \\
\text { (no. of pts) }\end{array}$} & \multicolumn{2}{|c|}{$\begin{array}{l}\text { Absence of AC cells } \\
\text { (\% of pts) }\end{array}$} & \multicolumn{2}{|c|}{$\begin{array}{l}\text { Absence of pain } \\
\text { (\% of pts) }\end{array}$} & \multicolumn{2}{|c|}{$\begin{array}{l}\text { Absence of AC flare } \\
\text { (\% of pts) }\end{array}$} & \multicolumn{2}{|c|}{$\begin{array}{l}\text { Pts requiring } \mathrm{RM} \\
\text { (\% of pts) }\end{array}$} \\
\hline & & Day 8 & Day 14 & Day 8 & Day 14 & Day 8 & Day 14 & Day 8 & Day 14 \\
\hline \multirow[t]{2}{*}{ OTX-13-002 [12, 17] } & DEX (164) & 13.5 & $33.1 * * a$ & $80.4 * * * a$ & $79.6 * * *$ & $52.1 * *$ & $71.6 * * *$ & $7.4 *$ & $14.7 * * *$ \\
\hline & PL (83) & 11.0 & $14.5^{\mathrm{a}}$ & $43.4^{\mathrm{a}}$ & 39.8 & 32.9 & 36.1 & 28.9 & 46.3 \\
\hline \multirow[t]{2}{*}{ OTX-14-003 [12, 17] } & $\operatorname{DEX}(161)$ & 22.5 & $39.4^{\mathrm{a}}$ & $77.5^{* * a, b}$ & $76.9^{* * \mathrm{~b}}$ & $63.1 * b$ & $66.3 * * \mathrm{~b}$ & $6.3 * * \mathrm{~b}$ & $17.5^{* * \mathrm{~b}}$ \\
\hline & PL (80) & 13.8 & $31.3^{\mathrm{a}}$ & $58.8^{\mathrm{a}}$ & 57.5 & 46.3 & 48.8 & 18.8 & 37.5 \\
\hline \multirow[t]{2}{*}{ OTX-15-003 [13] } & $\operatorname{DEX}(216)$ & $29.1 * * *$ & $52.3 * * * a$ & $79.6 * * * a$ & $84.2 * *$ & $58.2 * * *$ & $73.5 * * *$ & 3.3 & 5.6 \\
\hline & PL (222) & 11.7 & $31.1^{\mathrm{a}}$ & $61.3^{\mathrm{a}}$ & 70.1 & 36.9 & 49.3 & 4.1 & 10.9 \\
\hline \multirow[t]{2}{*}{ Pooled [20] } & DEX (539) & $22.4^{*}$ & $42.7 *$ & $79.2 *$ & $80.6^{*}$ & NR & NR & 5.4 & 11.7 \\
\hline & PL (385) & 12.0 & 27.5 & 56.9 & 60.9 & NR & NR & 12.5 & 24.0 \\
\hline
\end{tabular}

Analyses were performed using the intent to treat populations, and missing data were imputed for the primary endpoints (last observation carried forward)

$A C$ anterior chamber, $D E X$ dexamethasone intracanalicular insert, $N R$ not reported, $P L$ placebo, $p t s$ patients, $R M$ rescue medication

${ }^{*} p \leq 0.05, * * p<0.01, * * * p<0.0001$ vs PL

${ }^{\mathrm{a}}$ Co-primary endpoints

${ }^{b}$ Nominal $p$ values 
Patients were generally satisfied with the dexamethasone intracanalicular insert during OTX-15-003. A non-validated questionnaire administered during the last clinic visit (day 45 ) reported generally high satisfaction scores for the relief of eye pain (mean score 6.07 dexamethasone intracanalicular insert vs 5.43 placebo; $p<0.0001$ ) and eye discomfort (6.04 vs 5.33; $p<0.0001)$ on a $1-7$ scale, with higher scores indicating greater satisfaction. Similar satisfaction scores were reported for the treatment of eye pain (6.10 vs 5.34; $p<0.0001)$ and eye discomfort (5.95 vs 5.30; $p<0.0001)$. The overall satisfaction (4.31 vs $3.87 ; p<0.005)$, comfort (4.35 vs 4.09; $p<0.005$ ) and convenience (4.46 vs 4.15; $p<0.005)$ scores also indicated high satisfaction with the dexamethasone intracanalicular insert (assessed on a 1-5 scale, where 5 indicates highest satisfaction) [18]. These results were consistent with a small, non-validated survey $(n=25)$ of patients from OTX-13-002 and OTX-14-003 in which 23 of 25 patients were very satisfied with the dexamethasone intracanalicular insert. The survey also revealed that 22 of 23 patients found the dexamethasone intracanalicular insert to be very or extremely convenient in comparison with eye drops administered several times a day [19]. However, patient satisfaction scores should be interpreted with caution, as the clinical significance is unknown.

\subsection{Pooled Analyses}

The efficacy of the dexamethasone intracanalicular insert for the treatment of post-surgical ocular pain and inflammation was also demonstrated in a post hoc pooled analysis of the phase III trials (Table 2). Both co-primary endpoints for pain on day 8 and inflammation on day 14 were met (Table 2). The dexamethasone intracanalicular insert had a significant ( $p \leq 0.05$ ) effect on the percentage of patients with the absence of pain on days 2-30, as well as the absence of AC cells on days 4-30 [20]. The use of rescue medications was comparable across the groups on day 2 (1.1\% dexamethasone intracanalicular insert and $0.8 \%$ placebo) and day 4 (1.9\% and $2.3 \%$ ) [20], and half as many patients in the dexamethasone intracanalicular insert treatment group required rescue therapy on days 8 and 14 (Table 2).

In a subgroup of patients who required cataract surgery in the second eye $(n=292)$, the dexamethasone intracanalicular insert was effective in both first-eye and second-eye surgeries. A significantly higher $(p \leq 0.05)$ proportion of patients in the dexamethasone intracanalicular insert than placebo groups achieved an absence of pain on day 8 for both firsteye surgery $(80.5 \%$ vs $57.6 \%)$ and second-eye surgery $(76.2 \%$ vs $55.5 \%)$. Inflammation was also controlled by the dexamethasone intracanalicular insert, with a significantly $(p \leq 0.05)$ higher percentage of patients in the dexamethasone intracanalicular insert than placebo groups having an absence of AC cells on day 14 for both first-eye surgery
(44.0\% vs $27.2 \%$ ) and second-eye surgery (39.6\% vs $28.1 \%$ ) [15]. Therefore, the dexamethasone intracanalicular insert is likely to be effective in both subsets of patients.

\section{Tolerability of the Dexamethasone Insert}

The dexamethasone intracanalicular insert was generally well tolerated in a pooled analysis of a phase II trial and the phase III trials discussed in Sect. $3(n=567)$. The most common ocular adverse reactions that occurred during treatment with the dexamethasone intracanalicular insert were AC inflammation including iritis and iridocyclitis (10\% incidence), increased IOP (6\%), reduced visual acuity (2\%), cystoid macular oedema (1\%), corneal oedema (1\%), eye pain $(1 \%)$ and conjunctival hyperaemia (1\%), while headache was the most common non-ocular adverse reaction (1\%) [6]. Two patients $(0.4 \%)$ from the dexamethasone intracanalicular insert treatment groups and two patients $(0.5 \%)$ from the placebo groups withdrew from the trials due to an adverse event (AE) [21]. AEs were generally mild to moderate in severity, with four treatment-related AEs reported in dexamethasone intracanalicular insert groups [increased lacrimation $(n=2)$, eyelid irritation and increased IOP ( $n=1$ for both)] and four reported in placebo groups [cellulitis, conjunctivitis, dacryocanaliculitis and eye disorder ( $n=1$ for each)] [21].

Similar to other corticosteroid treatments [22], increases in IOP may occur with the dexamethasone intracanalicular insert [6]. Monitoring IOP is recommended during treatment with the dexamethasone intracanalicular insert, and caution is advised if the dexamethasone intracanalicular insert is used in patients with glaucoma [6]. During clinical trials, only 1 case from 38 cases of increased IOP (defined as $\geq 10 \mathrm{mmHg}$ ) was considered treatment-related in the dexamethasone treatment groups; most cases were attributed to the cataract surgery. Removal of the dexamethasone intracanalicular insert due to elevated IOP was not required in any patients, and in 16 cases of IOP elevation, no intervention was required [23]. A numerical increase in mean IOP was noted across the dexamethasone intracanalicular insert treatment groups in phase III trials on day 2 (day 1 post-operation; $+1.7 \mathrm{mmHg}$ on day 2 vs baseline), in addition to a significantly higher incidence of IOP AEs on day 2 compared with placebo $(6.3 \%$ and $2.3 \% ; p=0.0042)$. However, the mean IOP and the incidence of IOP AEs in the dexamethasone intracanalicular insert groups were generally comparable to placebo groups on subsequent visits (days 4-30) [23].

As dexamethasone is an immunosuppressive corticosteroid (Sect. 2), ocular infections may occur. The use of the dexamethasone intracanalicular insert is contraindicated in patients with active ocular infections, including infections 
of the cornea, conjunctiva or canaliculus [6]. However, no treatment-related infections were observed during treatment with the dexamethasone intracanalicular insert during phase III trials [21].

\section{Dosage and Administration of the Dexamethasone Insert}

One insert containing $0.4 \mathrm{mg}$ of dexamethasone is the recommended dosage for the treatment of ocular inflammation and pain following ophthalmic surgery, according to the US prescribing information. The dexamethasone intracanalicular insert is placed below the punctal opening of the lower lacrimal canaliculus (Fig. 2 illustrates the position of the insert), where it releases dexamethasone for up to 30 days. Manual removal of the dexamethasone intracanalicular insert is not necessary as the insert is resorbed, however, the dexamethasone intracanalicular insert may be manually expressed or irrigated with saline if removal is required [6]. Consult local prescribing information for detailed instructions regarding insertion, use in specific populations, warnings and precautions.

\section{Current Status of the Dexamethasone Intracanalicular Insert for Post-surgical Ocular Pain and Inflammation}

Currently available topical treatments for post-surgical pain and/or inflammation are corticosteroids (e.g. dexamethasone, loteprednol etabonate, difluprednate and prednisolone acetate [3]) and NSAIDs (e.g. bromfenac, nepafenac, diclofenac and ketorolac [24]). In addition to topical treatments, an injectable dexamethasone intraocular suspension is indicated for post-surgical inflammation [25]. The American Academy of Ophthalmology does not recommend a specific regimen for the control of post-surgical ocular pain and inflammation following cataract surgery, as an optimal regimen is yet to be ascertained with controlled studies. Best practice is to individualize treatments to meet the specific needs of patients [26]. This lack of evidence was also apparent in a meta-analysis of randomized, controlled trials for ocular inflammation following cataract surgery, which could not establish the relative efficacy of topical NSAIDs compared with topical corticosteroids [27]. Post-surgical regimens vary between practitioners [26]. In a worldwide survey of surgeons, 50\% of the respondents prescribed NSAIDs for 4 weeks and $73 \%$ prescribed a corticosteroid for 4 weeks following cataract surgery [28].

The dexamethasone intracanalicular insert is indicated for the treatment of post-surgical ocular pain and inflammation (Sect. 5). The insert is placed into the lower canaliculus via the lacrimal punctum (Fig. 2), where it releases a tapered dose of dexamethasone over 30 days (Sect. 2). Due to the extendedrelease profile of the dexamethasone intracanalicular insert, only one dose is required for the entire duration of treatment (Sect. 5). In contrast, the indicated dosing frequencies for topical treatments range from once daily (e.g. bromfenac $0.07 \%$ or $0.09 \%$ solution [24]) to four times daily (e.g. ketorolac $0.4 \%$ or $0.5 \%$ solution [24] or loteprednol etabonate $0.5 \%$ suspension [3]). This difference in dosing frequency may explain the strong patient preference for the dexamethasone intracanalicular insert over eye drops (Sect. 3). Additionally, as patient nonadherence with topical ocular therapies is a contributor to treatment failure [3], the one-time administration and tapered drug delivery of the dexamethasone intracanalicular insert may potentially improve clinical outcomes [12]. Although the intraocular dexamethasone suspension has a similar dosing frequency as the dexamethasone intracanalicular insert (one injection during surgery), the dexamethasone suspension is not currently indicated for post-surgical pain [25]. Furthermore, removal of the intraocular dexamethasone suspension was reported to be difficult [29], whereas the dexamethasone intracanalicular insert was easy or moderately easy to remove (when required) during phase III trials. In most cases, manual expression of the dexamethasone intracanalicular insert was not required as the insert is resorbed (Sect. 2).

The efficacy of the dexamethasone intracanalicular insert for the treatment of post-surgical ocular pain was demonstrated in three phase III trials, and the co-primary endpoint for post-surgical inflammation was met in two of these trials (Sect. 3). The result for the post-surgical inflammation coprimary endpoint in OTX-14-003 could be explained by the concomitant use of systemic NSAIDs (up to $40 \%$ of patients were taking systemic NSAIDs, including $\geq 1000 \mathrm{mg}$ naproxen per day in two patients in the placebo group) which may have masked the efficacy of the dexamethasone intracanalicular insert [12]. Of note, systemic NSAIDs were restricted to $375 \mathrm{mg}$ /day in the subsequent OTX-15-003 trial, which achieved both pain and inflammation co-primary endpoints [13]. The efficacy of the dexamethasone intracanalicular insert for post-surgical inflammation was supported by secondary endpoints, such as AC flare, in addition to pooled phase III trial data, which indicated that both co-primary endpoints were met (Sect. 3.1). A potential limitation of the phase III trials is the lack of an active comparator, and a noninferiority trial comparing the dexamethasone intracanalicular insert with a topical corticosteroid may have been a more appropriate design than a placebo-controlled trial [30]. However, the investigators noted that the trial design was acceptable to regulatory authorities, and outcomes in an active-comparator trial may be confounded by higher adherence rates with the topical corticosteroid compared with real-world clinical 
scenarios [30]. Therefore, an observational study of real-world outcomes may be valuable to address this concern.

Additional trials investigating the efficacy of the dexamethasone intracanalicular insert in other ocular surgeries, such as those to manage glaucoma or correct refractive errors, would be of interest. In particular, dry eye may occur following laser-assisted in situ keratomileusis (LASIK) surgery and occlusion of the punctal opening is associated with symptomatic relief [31]. The release of dexamethasone may complement this mechanism, as occlusion of the punctal opening does not treat the underlying inflammation associated with dry eye [32].

The dexamethasone intracanalicular insert was generally well tolerated, with $<1 \%$ of patients withdrawing because of AEs in an integrated analysis of phase II and III trials (Sect. 4). Although elevated IOP is associated with use of topical corticosteroids [22], increases in IOP AEs with the dexamethasone intracanalicular insert were limited to day 2 (i.e. 1 day after surgery). Additionally, most cases of elevated IOP were attributed to the cataract surgery and removal of the dexamethasone intracanalicular insert was not required in any patients due to elevated IOP (Sect. 4).

Overall, the dexamethasone intracanalicular insert was effective and generally well tolerated for the treatment of post-surgical ocular pain and inflammation. As low adherence is an issue for topical ocular corticosteroids, the single application of dexamethasone intracanalicular insert without the need for patient input makes it a promising emerging treatment option for inflammation and pain following ophthalmic surgery.

Data Selection Dexamethasone Intracanalicular Insert: 114 records identified

Duplicates removed

13

Excluded during initial screening (e.g. press releases; news reports; not relevant drug/indication; preclinical study; reviews; case reports; not randomized trial)

Excluded during writing (e.g. reviews; duplicate data; small patient number; nonrandomized/phase I/II trials)

Cited efficacy/tolerability articles

Search Strategy: EMBASE, MEDLINE and PubMed from 1946 to present. Clinical trial registries/databases and websites were also searched for relevant data. Key words were DEXTENZA, intracanalicular dexamethasone. Records were limited to those in English language. Searches last updated 19 May 2020
Acknowledgements During the peer review process, the manufacturer of the dexamethasone intracanalicular insert was also offered an opportunity to review this article. Changes resulting from comments received were made on the basis of scientific and editorial merit.

\section{Compliance with Ethical Standards}

Funding The preparation of this review was not supported by any external funding.

Conflict of interest Arnold Lee and Hannah Blair are salaried employees of Adis International Ltd/Springer Nature, are responsible for the article content and declare no relevant conflicts of interest.

Open Access This article is licensed under a Creative Commons Attribution-NonCommercial 4.0 International License, which permits any non-commercial use, sharing, adaptation, distribution and reproduction in any medium or format, as long as you give appropriate credit to the original author(s) and the source, provide a link to the Creative Commons licence, and indicate if changes were made. The images or other third party material in this article are included in the article's Creative Commons licence, unless indicated otherwise in a credit line to the material. If material is not included in the article's Creative Commons licence and your intended use is not permitted by statutory regulation or exceeds the permitted use, you will need to obtain permission directly from the copyright holder. To view a copy of this licence, visit http://creativecommons.org/licenses/by-nc/4.0/.

\section{References}

1. Schein OD, Cassard SD, Tielsch JM, et al. Cataract surgery among Medicare beneficiaries. Ophthalmic Epidemiol. 2012;19(5):257-64.

2. American Academy of Ophthalmology. Eye Health Statistics. https://www.aao.org/. Accessed 19 May 2020.

3. Salinger CL, Gaynes BI, Rajpal RK. Innovations in topical ocular corticosteroid therapy for the management of postoperative ocular inflammation and pain. Am J Manag Care. 2019;25(12 Suppl):S215-S226226.

4. Wu S, Tong N, Pan L, et al. Retrospective analyses of potential risk factors for posterior capsule opacification after cataract surgery. J Ophthalmol. 2018;2018:1-7.

5. Stone JL. An objective evaluation of eyedrop instillation in patients with glaucoma. Arch Ophthalmol. 2009;127(6):732.

6. Ocular Therapeutix. DEXTENZA ${ }^{\circledR}$ (dexamethasone ophthalmic insert) $0.4 \mathrm{mg}$, for intracanalicular use: US prescribing information. 2019. https://www.dextenza.com/. Accessed 19 May 2020.

7. Rhen T, Cidlowski JA. Antiinflammatory action of glucocorticoids-new mechanisms for old drugs. N Engl J Med. 2005;353(16):1711-23.

8. Center for Drug Evaluation and Research. Clinical pharmacology and biopharmaceutics review(s) (208742Orig1s000). 2016. https ://www.accessdata.fda.gov/. Accessed 19 May 2019.

9. Bafna S, Wortz G, Walters T, et al. Retention of Dextenza (dexamethasone intracanalicular insert), a sustained-release corticosteroid in phase 3 clinical trial program [abstract no. PO003 plus presentation]. In: American Academy of Ophthalmology Annual Meeting. 2019.

10. McGrath M, Blizzard CD, Desai A, et al. In vivo drug delivery of low solubility drugs from biodegradable hydrogel punctum plugs [abstract plus poster]. Investig Ophthalmol Vis Sci. 2014;55(13):472. 
11. Vanslette A, McGrath M, O'Connor M, et al. Dose based pharmacokinetics of sustained release dexamethasone in beagles [abstract plus poster]. Investig Ophthalmol Vis Sci. 2015;56(7):238.

12. Walters T, Bafna S, Vold S, et al. Efficacy and safety of sustained release dexamethasone for the treatment of ocular pain and inflammation after cataract surgery: results from two phase 3 studies. $\mathbf{J}$ Clin Exp Ophthalmol. 2016;7(4):1-11.

13. Tyson SL, Bafna S, Gira JP, et al. Multicenter randomized phase 3 study of a sustained-release intracanalicular dexamethasone insert for treatment of ocular inflammation and pain after cataract surgery. J Cataract Refract Surg. 2019;45(2):204-12.

14. Walters T, Endl M, Elmer TR, et al. Sustained-release dexamethasone for the treatment of ocular inflammation and pain after cataract surgery. J Cataract Refract Surg. 2015;41(10):2049-59.

15. Hovanesian JA, Tyson SL, Walters T, et al. Differences in postsurgical pain and inflammation outcome of patients receiving dexamethasone insert (Dextenza) based on fellow eye lens status [abstract no. PA024 plus presentation]. In: American Academy of Ophthalmology Annual Meeting. 2019.

16. Center for Drug Evaluation and Research. Statistical review(s) (208742Orig1s000). 2016. https://www.accessdata.fda.gov/. Accessed 19 May 2020.

17. Ocular Therapeutix. Data on file. 2015.

18. Noecker R, Hovanesian JA, Walters T, et al. Evaluating the physician and patient experience of a dexamethasone insert $(0.4 \mathrm{mg})$ in patients having cataract surgery [oral presentation]. In: American Society of Cataract and Refractive Surgery. 2018.

19. Gira JP, Sampson R, Silverstein SM, et al. Evaluating the patient experience after implantation of a $0.4 \mathrm{mg}$ sustained release dexamethasone intracanalicular insert (Dextenza): results of a qualitative survey. Patient Prefer Adherence. 2017;11:487-94.

20. Tyson SL, Bafna S, Berdahl JP, et al. Management of ocular inflammation and pain following cataract surgery with $0.4 \mathrm{mg}$ dexamethasone insert [abstract plus presentation]. In: American Society of Cataract and Refractive Surgery/American Society of Ophthalmic Administrators Annual Meeting. 2019

21. Hovanesian JA, Berdahl JP, Mah FS, et al. Integrated analysis of a dexamethasone insert $(0.4 \mathrm{mg})$ for the treatment of ocular pain after cataract surgery [abstract no. $\mathrm{PO} 295$ plus poster]. In: American Academy of Ophthalmology Annual Meeting. 2017.

22. Phulke S, Kaushik S, Kaur S, et al. Steroid-induced glaucoma: an avoidable irreversible blindness. J Curr Glaucoma Pract. 2017;11(2):67-72.

23. Vantipalli S, Metzinger JL, Goldstein MH. Intraocular pressure effects of DEXTENZA® (dexamethasone ophthalmic insert, 0.4 $\mathrm{mg}$ ), a novel sustained release steroid delivery via an intracanalicular insert, for treating post-cataract inflammation and pain [abstract plus presentation]. In: 3rd World Congress of Optometry. 2019
24. Hoffman RS, Braga-Mele R, Donaldson K, et al. Cataract surgery and nonsteroidal antiinflammatory drugs. J Cataract Refract Surg. 2016;42(9):1368-79.

25. EyePoint Pharmaceuticals. DEXYCU intraocular dexamethasone suspension: US prescribing information. 2018. https://dexyc u.com/. Accessed 19 May 2020.

26. Olson RJ, Braga-Mele $\mathrm{R}$, Chen $\mathrm{SH}$, et al. Cataract in the adult eye preferred practice pattern. Ophthalmology. 2017;124(2):P1-P119.

27. Juthani VV, Clearfield E, Chuck RS. Non-steroidal anti-inflammatory drugs versus corticosteroids for controlling inflammation after uncomplicated cataract surgery. Cochrane Database Syst Rev. 2017;7:CD010516.

28. American Society of Cataract and Refractive Surgery. ASCRS Clinical Survey 2017. https://supplements.eyeworld.org/. Accessed 19 May 2020.

29. Shah TJ, Conway MD, Peyman GA. Intracameral dexamethasone injection in the treatment of cataract surgery induced inflammation: design, development, and place in therapy. Clin Ophthalmol. 2018;12:2223-35.

30. Tyson SL, Bafna S, Gira JP, et al. Reply to: Grzybowski A, Kanclerz P. Benefits of noninferiority studies in ophthalmology. J Cataract Refract Surg. 2019;45(4):534-5.

31. Alfawaz AM, Algehedan S, Jastaneiah SS, et al. Efficacy of punctal occlusion in management of dry eyes after laser in situ keratomileusis for myopia. Curr Eye Res. 2013;39(3):257-62.

32. Tong L, Beuerman R, Simonyi S, et al. Effects of punctal occlusion on clinical signs and symptoms and on tear cytokine levels in patients with dry eye. Ocul Surf. 2016;14(2):233-41.

33. Esmailpour N, Högger P, Rohdewald P. Binding kinetics of budesonide to the human glucocorticoid receptor. Eur J Pharm Sci. 1998;6(3):219-23.

34. Blizzard C, Desai A, Driscoll A. Pharmacokinetic studies of sustained-release depot of dexamethasone in beagle dogs. J Ocul Pharmacol Ther. 2016;32(9):595-600.

35. McLaurin EB, Evans DG, Noecker R, et al. A single center study of the plasma pharmacokinetics of DEXTENZA (dexamethasone insert) $0.4 \mathrm{mg}$ in healthy volunteers [abstract no. 4106 plus poster]. Investig Ophthalmol Vis Sci. 2017;58(8):4106.

36. Ibach M, Metzinger JL, Vantipalli S, et al. Patient preference and physician experience with DEXTENZA (dexamethasone ophthalmic insert) $0.4 \mathrm{mg}$, a novel sustained-release intracanalicular insert for the treatment of postoperative pain and inflammation following cataract surgery [abstract plus poster]. In: 3rd World Congress of Optometry. 2019 